\title{
Intermediate Mass Black Holes and Nearby Dark Matter Point Sources: A Critical Reassessment
}

\author{
Torsten Bringmann \\ OKC, Department of Physics, Stockholm University, AlbaNova, SE - 10691 Stockholm - Sweden * \\ Julien Lavalle \\ Dipartimento di Fisica Teorica, Università di Torino \& INFN, via Giuria 1, 10125 Torino - Italy \\ Pierre Salati \\ LAPTH, Université de Savoie, CNRS, BP110, F-74941 Annecy-le-Vieux Cedex - Franc€
}

(Dated: 25 May 2009)

\begin{abstract}
The proposal of a galactic population of intermediate mass black holes (IMBHs), forming dark matter (DM) "mini-spikes" around them, has received considerable attention in recent years. In fact, leading in some scenarios to large annihilation fluxes in gamma rays, neutrinos and charged cosmic rays, these objects are sometimes quoted as one of the most promising targets for indirect DM searches. In this letter, we apply a detailed statistical analysis to point out that the existing EGRET data already place very stringent limits on those scenarios, making it rather unlikely that any of these objects will be observed with, e.g., the Fermi/GLAST satellite or upcoming Air Cherenkov telescopes. We also demonstrate that prospects for observing signals in neutrinos or charged cosmic rays seem even worse. Finally, we address the question of whether the excess in the cosmic ray positron/electron flux recently reported by PAMELA/ATIC could be due to a nearby DM point source like a DM clump or mini-spike; gamma-ray bounds, as well as the recently released Fermi cosmic ray electron and positron data, again exclude such a possibility for conventional DM candidates, and strongly constrain it for DM purely annihilating into light leptons.
\end{abstract}

PACS numbers: 95.35.+d, 97.60.Lf, 96.50.S

The nature of the mysterious DM, vastly dominating the total matter content of the universe, still remains unknown. Particularly plausible candidates, however, are weakly interacting massive particles (WIMPs) [1] and indirect DM searches aim at discriminating WIMP annihilation products from standard astrophysical backgrounds in gamma rays, neutrinos or charged cosmic rays.

Large DM density enhancements ("mini-spikes") around IMBHs have been proposed as promising targets for indirect DM searches in gamma rays [2], where a large number of very luminous point sources with identical cutoff in the photon spectrum would provide a smoking gun signature. Subsequent studies indicated excellent observational prospects also for neutrinos [3] and charged cosmic rays [4]. In the following, we restrict ourselves to IMBHs with a mass of around $10^{5} M_{\odot}$ that form out of collapsing cold gas in early-forming halos (scenario B of Ref. [2]), like in much of the literature on the subject, since only in this case one arrives at the above mentioned favorable prospects for indirect DM detection that have caused considerable recent attention.

While an application of our analysis to other IMBH formation scenarios would be straightforward, it is beyond the scope of this Letter, in which we critically re-assess the potential of IMBHs for DM searches. Our main conclusion is that the most favored DM parameter regions are actually already ruled out by the EGRET data [5] and that configurations predicting a signal in future searches - and yet being consistent with the existing constraints - are rather unlikely. For completeness, we will also derive generic limits on any nearby DM point-source.

Let us start by recalling some basic statistical properties of an ensemble of IMBHs in the galactic halo. Denoting with $P_{c}$ the probability for a particular IMBH to satisfy some condition $c$, the probability for $n$ out of $N$ objects to satisfy $c$ is given by

$$
P_{n, N}\left(P_{c}\right) \equiv\left(\begin{array}{c}
N \\
n
\end{array}\right) P_{c}^{n}\left(1-P_{c}\right)^{N-n} .
$$

Since the number of IMBHs in a given realization itself is a random variable with some distribution $p_{N}$ (in our case a Gaussian with mean $\sim 98$ and variance $\sim 21$ [4]), the probability that $n$ objects in an arbitrary realization satisfy $c$ becomes

$$
P_{n}^{\text {int }}\left(P_{c}\right) \equiv \int_{n}^{\infty} \mathrm{d} N p_{N}(N) P_{n, N}\left(P_{c}\right) .
$$

In our context, the relevant astrophysical properties of an IMBH are its distance $d$ to the Earth and, as a measure of the $\mathrm{DM}$ concentration, its annihilation volume $\xi \equiv \int \mathrm{d}^{3} x\left(\rho(\mathbf{x}) / \rho_{0}\right)^{2}$, where $\rho_{0}=0.3 \mathrm{GeV} \mathrm{cm}^{-3}$ is the local DM density. In particular, we will be interested in the probability that a single object has $\lambda=\xi / d^{2} \geq \lambda_{0}$ :

$$
P_{\lambda \geq \lambda_{0}}=\int_{0}^{\infty} \mathrm{d} \xi \int_{0}^{\left(\xi / \lambda_{0}\right)^{1 / 2}} \mathrm{~d} d\left(p_{\xi}(\xi) p_{d}(d)\right),
$$

where we use the (independent) probability densities $p_{d}$ and $p_{\xi}$ to find an IMBH at a distance $d$ from the earth 
and with a given annihilation volume $\xi$, respectively, from Ref. 4]. These distributions were obtained from Monte Carlo (MC) simulations that follow the evolution of initial mini-spike populations during their orbit in the Milky Way, taking account of possible close encounters. Armed with the above notation, we finally arrive at

$$
P_{n}^{\mathrm{cum}}\left(\lambda_{0}\right)=1-\sum_{i=0}^{n-1} P_{i}^{\mathrm{int}}\left(P_{\lambda \geq \lambda_{0}}\right)
$$

as the probability that a given realization contains at least $n$ objects with $\lambda \geq \lambda_{0}$.

The gamma-ray flux from a single IMBH is given by

$$
\Phi_{\mathrm{IMBH}}^{\gamma}=N_{\gamma} \Gamma /\left(4 \pi d^{2}\right),
$$

where $\Gamma=\frac{1}{2}\langle\sigma v\rangle\left(\rho_{0} / m_{\chi}\right)^{2} \xi$ is the local injection rate, $m_{\chi}$ the WIMP mass, $\langle\sigma v\rangle$ the annihilation rate (per unit density) and $N_{\gamma}$ the number of photons above some energy $E_{\min }$ per annihilation. At $E_{\gamma} \ll m_{\chi}$, the photon spectrum looks almost exactly the same for generic WIMP DM candidates that annihilate into quark or gauge boson final states; if not stated otherwise, we assume annihilation into $b \bar{b}$ and use PYTHIA [6] to compute $N_{\gamma}$. The adiabatic growth of an IMBH redistributes a typical initial DM density distribution into a steep profile (a "mini-spike") that is saturated in the innermost region due to DM self-annihilations and asymptotically develops an annihilation volume that scales like $\xi \propto\left(m_{\chi} /\langle\sigma v\rangle\right)^{5 / 7}$ [2]. Putting in numbers, Eq. (15) thus becomes

$$
\begin{aligned}
\Phi_{\mathrm{IMBH}}^{\gamma} & \approx 3.31 \cdot 10^{-7}\left(\frac{N_{\gamma}}{10}\right) \mathrm{cm}^{-2} \mathrm{~s}^{-1} \\
& \times\left(\frac{\tilde{\xi} / d^{2}}{10^{5} \mathrm{kpc}}\right)\left(\frac{m_{\chi}}{\mathrm{TeV}}\right)^{-\frac{9}{7}}\left(\frac{\langle\sigma v\rangle}{3 \cdot 10^{-26} \mathrm{~cm}^{3} \mathrm{~s}^{-1}}\right)^{\frac{2}{7}}
\end{aligned}
$$

where $\tilde{\xi}$ is the annihilation volume for an IMBH when $\langle\sigma v\rangle=3 \cdot 10^{-26} \mathrm{~cm}^{3} / \mathrm{s}$ and $m_{\chi}=1 \mathrm{TeV}$.

The upper limit on the flux from point sources not seen by the EGRET satellite strongly depends on the position on the sky, but is almost everywhere significantly below $\Phi_{\max }^{\infty} \sim 2 \times 10^{-7} \mathrm{~cm}^{-2} \mathrm{~s}^{-1}$ for $100 \mathrm{MeV} \lesssim E_{\gamma} \lesssim$ $30 \mathrm{GeV}$ [5]. On the other hand, a considerable number of detected sources with higher fluxes remains with no associated low-energy counterpart. Very conservatively assuming that all these unidentified sources are, in fact, connected to DM mini-spikes would translate into the requirement that at most $(1,4,10) \mathrm{IMBHs}$ have a flux larger than about $\Phi_{\max }^{(1,4,10)} \sim(7,5.5,3.7) \times 10^{-7} \mathrm{~cm}^{-2} \mathrm{~S}^{-1}$ [7]; as the spectra of these sources are in almost all cases much softer than what is expected from DM annihilation, however, this possibility does not appear to be very likely (note also that many of these EGRET sources are not confirmed by the first Fermi data [8]).

Demanding that at least $5 \%$ of the IMBH realizations should not be in conflict with the EGRET constraints

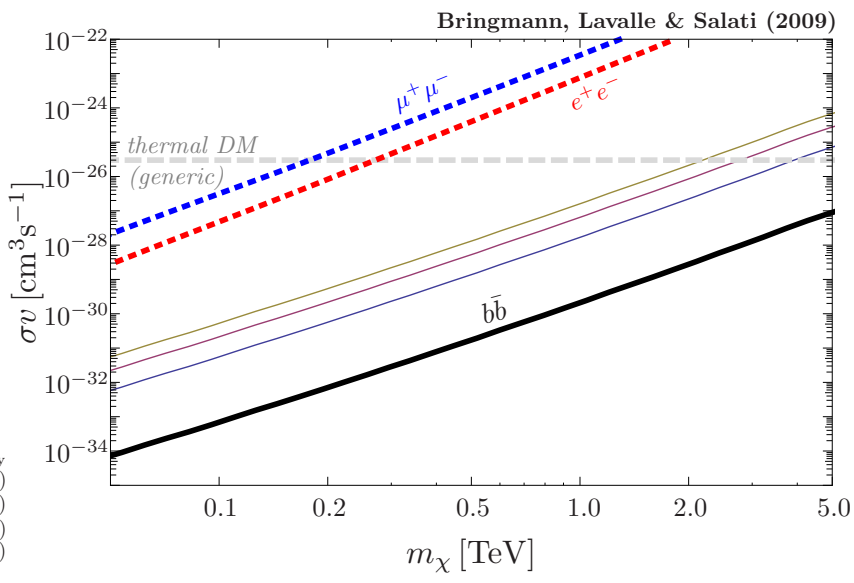

FIG. 1: The thin solid lines show the constraints on WIMPs in the IMBH scenario B of [2], from top to bottom deriving from the brightest 10, 4 and $1 \operatorname{object}(\mathrm{s})$, respectively. The region above the thick solid line is excluded by the EGRET limit on unresolved point sources; the dotted lines show the corresponding constraint if DM were to annihilate only into $e^{+} e^{-}$or $\mu^{+} \mu^{-}$. The dashed line indicates the canonical value of $\sigma v=3 \cdot 10^{-26} \mathrm{~cm}^{3} \mathrm{~s}^{-1}$ for thermally generated WIMPs.

on the $n$ brightest sources, we can now use Eq. (6) to derive bounds on the annihilation rate and mass of the annihilating particles by choosing $\lambda_{0}=\tilde{\xi} / d^{2}$ such that $P_{n}^{\text {cum }}\left(\lambda_{0}\right)>0.95$. The result is shown in Fig. 1 (for similar constraints from the H.E.S.S. experiment, see [9]). It is interesting to note that the $n \sim 10$ brightest unidentified EGRET sources lead to similar constraints. Having remarked before, however, that an IMBH interpretation of these objects is not too likely, we also include for comparison the much tighter constraint that results from considering the sensitivity limit of EGRET on unseen point sources. We verified these limits in extensive MC simulations and would like to draw special attention to the fact that they lie, indeed, several orders of magnitude below the expectation for generic WIMP candidates 1], $m_{\chi} \sim \mathcal{O}(100 \mathrm{GeV}-1 \mathrm{TeV})$ and $\langle\sigma v\rangle \sim \mathcal{O}\left(10^{-26} \mathrm{~cm}^{3} \mathrm{~s}^{-1}\right)$. Note that the constraints shown in Fig. 1 are, in fact even conservative as the EGRET limit on unresolved point sources is actually in many regions of the sky much smaller than the value used here.

The neutrino flux is simply obtained by replacing in Eq. (5) the index $\gamma$ with $\nu$, so the EGRET limit on gamma rays translates into $\Phi_{\text {IMBH }}^{\nu}<\left(N^{\nu} / N^{\gamma}\right) \Phi_{\max }^{\gamma}$. This limit on the neutrino flux has to be compared with the sensitivity of upcoming $\mathrm{km}^{3}$-sized neutrino telescopes [10]. Optimistically assuming an effective surface area for detection below $1 \mathrm{TeV}$ of $\sim 0.1\left(E_{\nu} / \mathrm{TeV}\right)^{2} \mathrm{~m}^{2}$, and using the Bartol model for the atmospheric neutrino background [11], we find a background rate of $\sim 10^{-4} \mathrm{~Hz}$. A $5 \sigma$ detection after 1 yr would then require a primary neutrino flux $\Phi_{\text {IMBH }}^{\nu} \gtrsim 10^{-8} \mathrm{~cm}^{-2} \mathrm{~s}^{-1}$ (over-optimistically assuming that the target is observed $100 \%$ of the time), 
which exceeds the above stated limit for typical values of $m_{\chi}$ and $N^{\nu}$. Of course, should DM annihilate mostly into neutrinos, the EGRET limit would be less stringent. However, even in conventional Kaluza-Klein (KK) scenarios [12], which offer large branching ratios to neutrinos, the EGRET constraint still excludes the observation of the neutrino counterpart with $\mathrm{km}^{3}$ detectors. Note also that large branching ratios into neutrinos actually mean smaller $N^{\nu}$, at least for large $m_{\chi}$, leading to overall worse prospects for detection [3].

Complementary to gamma rays, antiprotons are a further interesting channel of indirect DM detection [13]. To investigate whether the resulting constraints can compete with those from gamma rays in the mini-spike scenario, we have performed an extensive MC simulation of IMBH realizations. We found that antiprotons are competitive (i.e. in potential conflict with high-energy $\bar{p}$ data [14]) only for rather heavy WIMPs, with $m_{\chi} \gtrsim 1 \mathrm{TeV}$, and only when assuming extremely favorable propagation parameters, close to the max set defined in [15]; outside these somewhat extreme regions of the propagation parameter space, large $\bar{p}$ fluxes are generally obtained only in configurations that are already excluded by EGRET.

The excess in cosmic ray positrons and electrons recently reported by PAMELA [16], ATIC [17] and Fermi [18], if interpreted in terms of DM annihilation, points at $\mathrm{DM}$ masses in the $\mathrm{TeV}$ range with an unusually large branching ratio into light leptons [19]. In order to fit the data, however, standard DM candidates need extremely large boost factors that are not expected in current models of structure formation [20], corresponding to effective annihilation rates $\sim 10^{3}$ times the generic value for thermally produced DM. For comparison, we thus furthermore include in Fig. 1 the extreme situation of DM particles annihilating only into light leptons, in which case much less photons are produced and we can apply the analytic expression for $N_{\gamma}$ given in 21]; even in this somewhat contrived situation low-mass models turn out to be difficult to realize, at least for strongly enhanced annihilation rates, making it very unlikely for, e.g, Fermi-LAT 22] to see the expected cutoff in the photon spectrum for an IMBH that has escaped detection by EGRET.

Let us now turn to the possibility that the large required annihilation flux can be attributed to a nearby high DM concentration, like for example a DM clump or a DM mini-spike around an IMBH, located at some distance $d$ to the Earth. In Fig. 2, we use Eq. (5) and the EGRET sensitivity limit to constrain the annihilation rate $\Gamma$ of such a generic DM point source. In the same figure, we indicate as a gray area in the $\Gamma-d$ plane the annihilation rate that would be necessary to explain the PAMELA data, taking into account the allowed range for the $e^{+} / e^{-}$propagation parameters (consistent with both the background and the signal) by using the $\mathrm{min} / \mathrm{med} / \mathrm{max}$ configurations of [23]. The dark shaded region, finally, shows the rather conservative constraint on these combinations of $\Gamma$ and $d$ that arises from requiring that the positron and electron flux from DM annihilation alone should not exceed the Fermi data. For comparison, we separately consider the case of KK DM, as an example of a standard WIMP with exceptionally large branching ratios into light leptons, as well as DM only annihilating to $e^{+} e^{-}$or $\mu^{+} \mu^{-}$.

The first important observation is that standard WIMPs, which usually feature smaller branching ratios into light leptons than KK DM, basically cannot account for the PAMELA/ATIC data in this way without violating the EGRET bounds - the reason being the still relatively large contribution from non-leptonic channels to the photon spectrum at low energies. However, if one takes a more phenomenological approach and allows DM particles annihilating at $100 \%$ into $e^{+} e^{-}$or $\mu^{+} \mu^{-}$pairs, one would technically be able to fit the data by placing a DM point source at a distance $2 \mathrm{kpc} \lesssim d \lesssim 5 \mathrm{kpc}$ - at the price of requiring an enormously bright object, more luminous than the whole Milky Way! This would correspond to a DM clump of mass $\gtrsim 10^{11} M_{\odot}$ in conventional cosmological scenarios 24]. Since finding such a massive clump relatively close to the Earth is extremely unlikely [20], we arrive at a considerably more pessimistic conclusion than recently obtained by [25] about the possibility of explaining the PAMELA data in terms of a nearby clump of annihilating WIMPs. Mini-spikes, on the other hand, are extremely bright: for DM with $\langle\sigma v\rangle \sim 3 \cdot 10^{-26} \mathrm{~cm}^{3} \mathrm{~s}^{-1}$ in the IMBH scenario discussed earlier, e.g., the probability to encounter at least one object inside the light gray area of the middle (right) panel of Fig. 2 is roughly $84 \%$ (37\%). For a - certainly nonstandard - WIMP candidate annihilating almost exclusively into $e^{+} e^{-}$or $\mu^{+} \mu^{-}$, a galactic population of IMBHs might thus indeed provide a positron flux large enough to fit the data and yet be consistent with present-day constraints. Note, however, that this conclusion does not hold for DM candidates with intrinsically enhanced annihilation rates: already for $\langle\sigma v\rangle \sim 10^{-24} \mathrm{~cm}^{3} \mathrm{~s}^{-1}$, the above quoted probabilities drop to $0.9 \%(0.1 \%)$.

In this Letter, we have reconsidered the prospects for indirect DM detection in the presence of a galactic population of IMBHs and found them not to be very promising given that existing data, in particular from gamma rays, already place severe constraints on the scenario. While it was noted before that already EGRET should have seen some of these objects [2], the resulting constraints were not taken into account in the subsequent studies of, e.g., 3, 4]. This is the main reason for the discrepancy between our pessimistic and earlier rather optimistic conclusions about possible effects of DM mini-spikes on indirect DM searches. While beyond the scope of this letter, it would be interesting to extend the study presented here and apply a consistent treatment of all available constraints also to other IMBH formation scenarios in order to predict realistic prospects for indirect DM detection. 

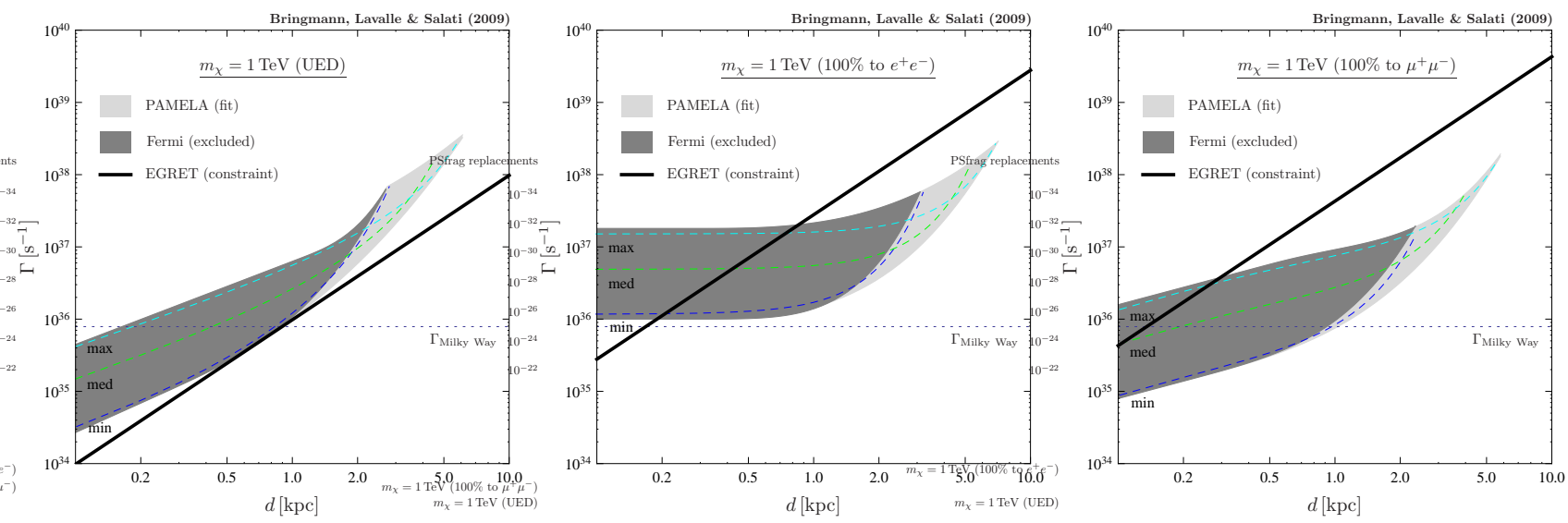

FIG. 2: The solid lines give the EGRET constraints on the DM annihilation rate $\Gamma=\frac{1}{2} \sigma v\left(\rho_{0} / m_{\chi}\right)^{2} \xi$ of a nearby, generic DM point-source at a distance $d$ from the Earth; from left to right, we show the case of KK DM and a fiducial DM candidate annihilating to $e^{+} e^{-}$and $\mu^{+} \mu^{-}$, respectively. The dashed lines show the $\Gamma$ needed to fit the PAMELA data, for sets of propagation parameters as defined in [23]; in the dark shaded area this would produce an $e^{ \pm}$flux in conflict with the Fermi data at higher energies. For comparison, the dotted line indicates $\Gamma$ for the whole Milky Way, assuming $\langle\sigma v\rangle \sim 3 \cdot 10^{-26} \mathrm{~cm}^{-3} \mathrm{~s}^{-1}$.

We have also addressed the possibility of explaining the recent PAMELA observations in terms of DM annihilation by placing a dark object in close vicinity to the Earth. While this option is ruled out from the gammaray constraints for standard WIMP candidates, a nearby IMBH (but not an ordinary DM clump) may in principle provide a sufficient amount of positrons if one assumes that the DM particles annihilate purely into light leptons. Even this seemingly a bit far-fetched scenario could soon be ruled out if Fermi [22] does not observe any corresponding point-sources in gamma rays. We would like to use this opportunity to recall that a very plausible hypothesis for the PAMELA results is anyway a nearby pulsar, i.e. an explanation in terms of astrophysics rather than DM annihilation [26].

Acknowledgements: We greatly thank Gianfranco Bertone for letting us use his catalogue of IMBH sources and the anonymous referees for very useful suggestions. TB and JL thank LAPTH for hospitality when this work was initiated. This work is supported, in part, by the French ANR project ToolsDMColl (BLAN07-2-194882). PS thanks the Perimeter Institute for hospitality when this work was finished.

* Electronic address: troms@physto.se

$\dagger$ Electronic address: lavalle@to.infn.it

¥ Electronic address: salati@lapp.in2p3.fr

[1] G. Jungman, M. Kamionkowski and K. Griest, Phys. Rept. 267, 195 (1996); L. Bergström, Rept. Prog. Phys. 63, 793 (2000); G. Bertone, D. Hooper and J. Silk, Phys. Rept. 405, 279 (2005).

[2] G. Bertone, A. R. Zentner and J. Silk, Phys. Rev. D 72, 103517 (2005). M. Fornasa and G. Bertone, Int. J. Mod.
Phys. D 17, 1125 (2008).

[3] G. Bertone, Phys. Rev. D 73, 103519 (2006).

[4] P. Brun et al., Phys. Rev. D 76 (2007) 083506.

[5] Hartman et al., Ap.J.S. 123, 79 (1999).

[6] T. Sjöstrand, S. Mrenna and P. Skands, JHEP 0605, 026 (2006) arXiv:hep-ph/0603175.

[7] V. Pavlidou et al., Astrophys. Space Sci. 309 (2007) 81.

[8] A. A. Abdo et al. arXiv:0902.1340 [astro-ph.HE].

[9] F. Aharonian et al., Phys. Rev. D 78, 072008 (2008).

[10] See, e.g., T. Montaruli, arXiv:0901.2661 [astro-ph].

[11] G. Barr, T. K. Gaisser and T. Stanev, Phys. Rev. D 39 (1989).

[12] D. Hooper and S. Profumo, Phys. Rept. 453, 29 (2007).

[13] T. Bringmann and P. Salati, Phys. Rev. D 75, 083006 (2007); F. Donato et al., Phys. Rev. Lett. 102, 071301 (2009).

[14] O. Adriani et al., Phys. Rev. Lett. 102, 051101 (2009).

[15] F. Donato et al., Phys. Rev. D 69, 06351 (2004).

[16] O. Adriani et al., arXiv:0810.4995 [astro-ph].

[17] J. Chang et al., Nature 456, 362 (2008).

[18] A. A. Abdo et al. Phys. Rev. Lett. 102, 181101 (2009).

[19] see, e.g., M. Cirelli, M. Kadastik, M. Raidal and A. Strumia, Nucl. Phys. B 813, 1 (2009).

[20] V. Springel et al., MNRAS 391, 1685 (2008).

[21] L. Bergström, T. Bringmann, M. Eriksson and M. Gustafsson, Phys. Rev. Lett. 94, 131301 (2005).

[22] E. A. Baltz et al., JCAP 0807, 013 (2008).

[23] T. Delahaye et al., arXiv:0809.5268 [astro-ph].

[24] J. Lavalle, Q. Yuan, D. Maurin and X. J. Bi, Astron. Astrophys. 479, 427 (2008).

[25] D. Hooper, A. Stebbins and K. M. Zurek, arXiv:0812.3202 [astro-ph].

[26] A. Boulares, ApJ 342, 807 (1989); F. A. Aharonian, A. M. Atoyan and H. J. Voelk, Astron. Astrophys. 294, L41-L44 (1995); H. Yüksel, M. D. Kistler and T. Stanev, arXiv:0810.2784 D. Hooper, P. Blasi and P. D. Serpico, JCAP 0901, 025 (2009); S. Profumo, N. J. Shaviv, E. Nakar and T. Piran, arXiv:0902.0376 [astro-ph.HE]. 